\title{
Influência da dor crônica na qualidade de vida em idosos*
}

\section{Influence of chronic pain in the quality of life of the elderly}

\author{
Lorena Lourenço Cunha ${ }^{1}$, Wildete Carvalho Mayrink²
}

* Recebido do Curso de Medicina do Centro Universitário do Maranhão. São Luís, MA.

- Trabalho de Conclusão do Curso de Medicina do Centro Universitário do Maranhão.

\section{RESUMO}

JUSTIFICATIVA E OBJETIVOS: A dor crônica é um dos problemas mais importantes, que dificultam consideravelmente a autonomia do idoso no desempenho de suas funções diárias, afetando sua qualidade de vida (QV). Os objetivos deste estudo foram avaliar a influência da dor crônica na QV dos idosos, usando a escala analógica visual (EAV) da dor e o questionário World Health Organization Quality of Life (WHOQOL-bref), observando suas repercussões nos quatro domínios: físico, psicológico, relações sociais e meio ambiente.

MÉTODO: Realizou-se este estudo transversal descritivo, exploratório com uma amostragem de conveniência composta por 50 idosos, com diagnóstico clínico de dor crônica e idade igual ou superior a 60 anos. Foi utilizado como Instrumento de Avaliação, o questionário WHOQOL-bref e a EAV de Dor.

RESULTADOS: Foram identificados 34\% com distúrbios da coluna; $22 \%$ com artrite reumatoide, $18 \%$ com osteoporose, $14 \%$ com artrose e $12 \%$ com tendinopatias. Pela EAV foi identificado padrão de dor moderada. Houve uma influência negativa da dor crônica na QV dos idosos

1. Acadêmica do Curso de Medicina do Centro Universitário do Maranhão, São Luís, MA, Brasil.

2. Médica Graduada pela Universidade Federal do Maranhão, Especialista em Clínica Médica pela Santa Casa de Misericórdia do Rio de Janeiro; Pós-Graduada em Acupuntura pela Sociedade Médica Brasileira de Acupuntura; Médica do Hospital Universitário Presidente Dutra; Professora do Curso de Medicina do Centro Universitário do Maranhão. São Luís, MA, Brasil.

Endereço para correspondência:

Lorena Lourenço Cunha

Rua Balsas Qd. 46/09 - Quintas do Calhau

65067-460 São Luís, MA.

Fones: (98) 8134-1070 - (98) 3226-2701

E-mail: lorelourenco@hotmail.com

Telemóvel: 351967080994

E-mail: angela.simoes@gmail.com no domínio físico e nas relações sociais em relação aos voluntários controles.

CONCLUSÃO: Este estudo evidenciou a presença de dor moderada avaliada pela escala unidimensional, com influências negativas na QV dos idosos, e os profissionais de saúde devem estar sempre atentos e utilizar métodos avaliativos que possam identificar a magnitude do quadro álgico, uma vez que muitas vezes o idoso não saber expressar adequadamente sua dor, para oferecer ao idoso todo o suporte de saúde necessário.

Descritores: Crônica, Dor, Idoso, Qualidade de vida.

\section{SUMMARY}

BACKGROUND AND OBJECTIVES: Chronic pain is one of the most important problems considerably impairing autonomy of the elderly to perform their daily functions, highly impairing their quality of life (QL). This study aimed at evaluating the influence of chronic pain on the QL of the elderly, using the pain visual analog scale (VAS) and the World Health Organization Quality of Life (WHOQOL-bref) questionnaire, observing its repercussions on four domains: physical, psychological, social relations and environment.

METHOD: This was a transversal, descriptive, exploratory study with a convenience sample of 50 elderly people with clinical diagnosis of chronic pain, aged 60 years or above. Evaluation tools were the WHOQOL-bref questionnaire and the pain VAS.

RESULTS: We identified 34\% with spinal disorders; $22 \%$ with rheumatoid arthritis, $18 \%$ with osteoporosis, $14 \%$ with arthritis and $12 \%$ with tendon injuries. Pain was moderate according to the VAS. There has been a negative influence of chronic pain on the QL of the elderly in the physical domain and in social relations as compared to control voluntary people.

CONCLUSION: This study has shown the presence of 
moderate pain evaluated by the one-dimension scale, with negative influence on the QL of the elderly, and health professionals should be always attentive and should use evaluation methods which may identify the magnitude of pain, since very often the elderly do not know how to adequately explain their pain, to be able to offer the elderly all needed health support.

Keywords: Chronic, Elderly, Pain, Quality of life.

\section{INTRODUÇÃO}

A dor crônica acomete, em todo o mundo, cerca de 100 milhões de indivíduos e aproximadamente, $14 \%$ da dor crônica está relacionada às articulações e ao sistema musculoesquelético ${ }^{1}$.

A dor crônica, como doença e não sintoma pode ter consequências na qualidade de vida (QV). Fatores como depressão, incapacidade física e funcional, dependência, afastamento social, mudanças na sexualidade, alterações na dinâmica familiar, desequilíbrio econômico, desesperança, sentimento de morte e outros, encontram-se associados a quadros de dor crônica. A dor passa a ser o centro, direciona e limita as decisões e comportamentos do indivíduo. Acarreta, ainda, fadiga, anorexia, alterações do sono, constipação, náuseas, dificuldade de concentração, entre outros. A impossibilidade de controlá-la traz sempre sofrimento físico e psíquico. Todos esses fatores associados parecem aumentar a morbidade entre os idosos e onerar o sistema de saúde².

A boa QV auxilia a manutenção da autonomia do idoso. Uma forma de quantificá-la é associá-la ao grau de autonomia que o idoso desempenha as suas funções no dia a dia, tornando-o independente dentro de um contexto social, econômico e cultural. A QV na velhice não é um atributo do indivíduo biológico, psicológico ou social, nem uma responsabilidade individual, mas sim, um produto da interação entre as pessoas vivendo numa sociedade em mudanças ${ }^{3}$.

Ainda permanece carente a investigação sobre a aparente contradição entre velhice e bem estar, ou mesmo a associação entre velhice e dor crônica e sobre as condições que permitem uma boa QV na velhice. O tema tem grande importância científica e social inclusive para a compreensão do envelhecimento e a criação de alternativas de intervenção visando o bem estar de pessoas idosas ${ }^{4}$.

Devido à carência de dados, somada a importância do tema, o objetivo deste estudo foi avaliar as condições de vida dos idosos num âmbito multidisciplinar, para avaliar a influência da dor crônica na QV dos idosos.

\section{MÉTODO}

Após aprovação do projeto pela Comissão Científica do Centro Universitário do Maranhão e pelo Comitê de Ética em pesquisa da mesma instituição sob o protocolo $n^{\circ}$ 00380/10, realizou-se este estudo transversal no período de outubro de 2010 a fevereiro de 2011, com pacientes do serviço ambulatorial de acupuntura de uma Unidade Mista de Saúde de São Luís-MA. Todos os pacientes tinham o diagnóstico clínico de dor crônica e idade igual ou superior a 60 anos.

A amostragem foi de conveniência e o cálculo do seu tamanho foi realizado com base nos quatro domínios do instrumento World Health Organization Quality of Life (WHOQOL), utilizado na coleta de dados, com um desvio de $10 \%$ em cada domínio. O tamanho da amostra ficou estabelecido em 50 pacientes.

Não foram incluídos os pacientes com doenças que promovessem mais incapacidades que a dor crônica, tais como: pneumopatia ou cardiopatia grave, doença neurológica ou oncológica e depressão.

Foram utilizados o Instrumento de Avaliação de QV da Organização Mundial de Saúde, o WHOQOL-bref, versão em português falado no Brasil e a escala analógica visual (EAV).

Os pacientes foram informados sobre os objetivos do estudo a confidencialidade dos dados e assinaram o termo de consentimento livre e esclarecido. Todos os pacientes responderam o WHOQOL-bref em um encontro, referindo-se à situações ocorridas nas duas semanas anteriores.

Este instrumento, a princípio, é de autorresposta, administrado pelo entrevistador, não influenciando o participante na escolha da resposta. As questões ou o significado destas, assim como a escala de respostas, não foram discutidas. Em caso de dúvida, o entrevistador apenas releu a questão de forma pausada, evitando dar sinônimos às palavras das perguntas. Foi enfatizada a importância da interpretação da pergunta, pelo paciente. Ao término do questionário, foi verificado se o paciente não havia deixado nenhuma questão sem resposta e se havia marcado somente uma alternativa por questão.

A pontuação do questionário foi efetuada pelo programa estatístico Statistical Package for the Social Sciences (SPSS- Windows 11.5).

Para a comparação entre os domínios e a média da normalidade, utilizou-se o teste $\mathrm{t}$ univariado. Para as correlações foi utilizada a Correlação Linear de Pearson. O nível de significância foi de $\mathrm{p}<0,05$. 


\section{RESULTADOS}

Foram estudados 50 pacientes sendo $76 \%$ mulheres, com média de idade de $72 \pm 7,36$ anos, sendo $38 \%$ casados, $28 \%$ viúvos, $22 \%$ solteiros e $12 \%$ separados. Quanto ao nível educacional $42 \%$ frequentaram apenas o ensino fundamental, $28 \%$ o ensino médio, $24 \%$ analfabetos e $6 \%$ fizeram o ensino superior.

As doenças mais referidas foram: artrite ou reumatismo em $62 \%$ dos pacientes, hipertensão arterial em $28 \%$ e diabetes em 10\% deles. Foram identificados 34\% dos pacientes com distúrbios da coluna, $22 \%$ com artrite reumatoide, $18 \%$ com osteoporose, $14 \%$ com artrose e $12 \%$ com tendinopatias.

Em relação à percepção subjetiva da saúde 6\% dos pacientes a classificaram como muito fraca, $30 \%$ como fraca, $52 \%$ nem ruim nem boa, $12 \%$ boa e nenhum a considerou como muito boa.

O índice de Cronbach mostrou valor elevado $(0,842)$ em todas as questões, confirmando a boa consistência interna do instrumento. A intensidade da dor foi moderada, sendo menor para as mulheres $(3,48 \pm 0,54)$ quando comparada com os homens $(4,31 \pm 1,09)$.

Não se identificou diferença significativa entre o nível educacional e os domínios, e entre o nível educacional e a representação de dor pela EAV. As mulheres apresentam maior satisfação no domínio: relações sociais, do que os homens.

Não foi observada correlação na percepção da própria saúde e a intensidade da dor pela EAV, mas houve correlação com o domínio físico $(0,53-\mathrm{p}<0,01)$, no domínio psicológico $(0,312-\mathrm{p}<0,05)$, no domínio meio ambiente $(0,325-\mathrm{p}<0,05)$ e nas perguntas gerais $(0,743$ $-\mathrm{p}<0,01)$.

Houve pequena correlação entre a intensidade de dor e o domínio físico de - 0,39 e entre o domínio psicológico de $-0,38$.

Os voluntários-controles $(16,61 \pm 2,1)$ apresentaram melhor QV no domínio físico que os idosos $(13,65 \pm 2,41)$; diferença estatisticamente significativa $(\mathrm{p} \leq 0,001)$.

No domínio psicológico não foi verificada diferença significativa entre o grupo voluntário-controle $(15,58 \pm 2,1)$ e os idosos $(15,04 \pm 2,32)$, porém no domínio relações sociais a diferença foi significativa $(\mathrm{p} \leq 0,02)$, pois o grupo voluntário-controle $(15,52 \pm 2,6)$ apresentou melhor QV que o grupo idoso $(14,39 \pm 3,15)$. No domínio meio ambiente também foi observada diferença significativa $(p \leq 0,001)$, uma vez que os idosos $(15,67 \pm 1,81)$ apresentaram melhor QV que os voluntários-controles $(14,04 \pm 2,1)$.

\section{DISCUSSÃO}

O conceito de saúde no idoso é abrangente e não se restringe à presença ou ausência de doença ou agravo e é estimada pelo nível de independência e autonomia. A avaliação deve ser multidimensional, levando-se em conta o bem estar biopsicossocial e a necessidade de ações integradas da equipe multidisciplinar ${ }^{5}$.

Vários métodos têm sido utilizados para mensurar a percepção/sensação de dor. Alguns consideram a dor como uma qualidade simples, unidimensional que varia apenas em intensidade, mas outros métodos a consideram como uma experiência multidimensional composta também por fatores afetivos e emocionais ${ }^{6}$.

As escalas de intensidade pertencem ao grupo de avaliação unidimensional, por avaliar somente uma das dimensões da experiência dolorosa, como a EAV, importante instrumento na mensuração da dor crônica, pois ajuda o paciente a compreender melhor quando e quais fatores influenciam a sua percepção da dor, mas devido suas limitações é muitas vezes pouco utilizada e/ou compreendida ${ }^{7-10}$.

Devido à complexidade para avaliar a QV, a Organização Mundial de Saúde (OMS) desenvolveu o World Health Organization Quality of Life (WHOQOL-100). A necessidade de instrumentos curtos que demandem pouco tempo para seu preenchimento, mas com características psicométricas satisfatórias, fez com que o Grupo de QV da Organização Mundial da Saúde desenvolvesse uma versão abreviada do WHOQOL-100 o WHOQOL ABREVIADO (WHOQOL-bref.) um questionário com 26 questões, composto por quatro domínios: físico, psicológico, relações sociais e meio ambiente ${ }^{11,12}$.

Suas questões são curtas e elaboradas em linguagem simples que compatibilizam com os quatro tipos de escala de avaliação estabelecidos (intensidade, capacidade, frequência e avaliação), com cinco níveis de respostas para cada uma. Essas escalas de respostas compreendem a escala de intensidade (nada a extremamente), capacidade (nada a completamente), frequência (nunca a sempre) e avaliação (muito insatisfeito a muito satisfeito, muito ruim a muito bom) ${ }^{13}$.

Os dados obtidos devem ser tratados em função de cada domínio, pois neste instrumento não se pode utilizar um escore total de QV, já que foi desenvolvido a partir da premissa de que QV é um construto multidimensional, não cabendo a soma de itens referentes a diferentes domínios ${ }^{13}$.

Como mostra a literatura este estudo evidenciou que a doença osteoarticular foi o fator que, mais frequentemente, causou dor nos idosos?. 
A fraca correlação nos domínio físico e psicológico e a ausência de correlação nos domínios relações sociais e o meio ambiente e a intensidade da dor avaliada pela EAV pode ser devida ao fato desse instrumento avaliativo ser unidimensional e avaliar apenas a intensidade de dor. Essa escala foi utilizada por ser um instrumento de fácil e rápida compreensão com o intuito de avaliar a intensidade da dor, que foi moderada ${ }^{13}$.

O WHOQOL-bref apresentou boa consistência interna, pelos valores observados pelo coeficiente $\alpha$ de Cronbach em todas as suas questões ${ }^{12}$.

$\mathrm{Na}$ ausência de estudos populacionais que padronizem cada item do WHOQOL-bref, os resultados obtidos neste estudo foram comparados com os do grupo de voluntários controles, provenientes da pesquisa de validação do referido questionário no Brasil, que apresentou poder amostral no processo de sua validação ${ }^{15}$.

Foi possível diferenciar idosos e indivíduos-controle nos domínios físico, relações sociais e meio ambiente, porém não no domínio psicológico, provavelmente por fatores referentes ao instrumento ou a amostra estudada. Por outro lado, é possível que o aspecto psicológico não esteja ainda necessariamente afetado pela condição da doença, quando comparado aos indivíduos-controle.

A QV, no aspecto meio ambiente foi superior nos idosos do que no grupo de voluntários-controles talvez devido aos critérios de inclusão utilizados.

Estudo semelhante realizado no Rio Grande do Sul, local onde se verificam melhores condições sociais, sanitárias, econômicas e culturais, razão no qual permite uma maior expectativa de vida pode-se comparar que numa amostra composta por 43 pacientes, em que $81,4 \%$ eram mulheres, com idade média de 72 anos, variando 60 a 90 anos, entre os problemas de saúde, os mais citados foram artrite ou reumatismo em $48,8 \%$ dos pacientes e $25,6 \%$ hipertensão arterial e dentre as alterações clínicas encontradas observou-se que $41,1 \%$ apresentaram artrose, $34,9 \%$ com distúrbio da coluna, 23,2\% com tendinopatias, 2,3\% com osteoporose e 2,3\% com artrite reumatoide. Não foram incluídos na pesquisa pacientes analfabetos e nenhum considerou a dor como muito fraca, porém houve diminuição na QV nos domínios físico e de relações sociais dos idosos ${ }^{15}$.

A aplicação do WHOQOL-bref permitiu evidenciar a influência negativa da dor crônica na QV no aspecto físico e nas relações sociais dos idosos estudados. Observou-se que a dor se associou à fadiga, aos distúrbios do sono, a dependência de tratamentos ou ao uso de medicamentos. Além de diminuir a capacidade para o trabalho, para a realização das atividades de vida diária, para a atividade sexual e se associa com a diminuição da rede de apoio social ${ }^{10}$.

A diminuição na $\mathrm{QV}$ nos domínio físico e de relações sociais dos idosos com dor crônica pode afetar também a manutenção da autonomia do idoso, o que poderá limitar o desempenho de suas atividades cotidianas, tornando-o dependente dentro de seu contexto social, econômico e cultural ${ }^{10}$.

O envelhecimento com QV depende do equilíbrio entre as limitações e as potencialidades do idoso. A probabilidade de envelhecer com boa QV aumentam em razão da adequada atuação dos serviços de saúde. Nesse processo de preparação e adaptação da sociedade a essa realidade demográfica, deve-se incluir a capacitação dos profissionais que terão a função de cuidar da saúde dos idosos, pois, atualmente, há uma carência significativa de profissionais com essa habilitação.

É fundamental a organização dos serviços em ações básicas de atenção à saúde do idoso na produção do cuidado em defesa da vida, pois para evitar orientações mal compreendidas pelos idosos e também a maior demanda nos serviços de saúde por não conseguirem julgar adequadamente sua dor, faz-se necessário o estudo constante, na área de QV no idoso e nos fatores que possam influenciá-la. Assim, será possível atuar preventivamente nas diversas doenças para que o idoso tenha melhores condições de preveni-las, para que continuem a ter autonomia no seu cotidiano.

Apesar de o WHOQOL-bref apresentar vocabulário simples e questões estruturadas para facilidade de sua leitura mesmo por pessoas com nível educacional baixo e com vocabulário restrito, é importante a utilização de um instrumento direcionado para a avaliação da QV para contemplar a população idosa, como o WHOQOL-OLD que tem como objetivo atuar melhor dentro de uma gama de critérios, além de acrescentar facetas adicionais aos instrumentos genéricos a fim de avaliar adequadamente a QV dos idosos. Isso porque, os idosos diferem das outras faixas etárias por constituírem um grupo com características particulares e, como tal, apresenta especificidades relevantes para a $\mathrm{QV}$.

\section{CONCLUSÃO}

Este estudo evidenciou a presença de dor moderada avaliada pela escala unidimensional, com influências negativas na QV dos idosos, e os profissionais de saúde devem estar sempre atentos e utilizar métodos avaliativos que possam identificar a magnitude do quadro álgico, uma vez que muitas vezes o idoso não saber expressar adequada- 
mente sua dor, para oferecer ao idoso todo o suporte de saúde necessário.

\section{AGRADECIMENTOS}

Aos médicos: Mércia Helena Salgado L. de Souza, Aldifran Ferreira da Silva, Daniel Nery de Oliveira pelo grande auxílio no desenvolvimento desta pesquisa.

\section{REFERÊNCIAS}

1. Gagliese L, Melzack R. Age- related differences in the qualities but not the intensity of chronic pain. Pain 2003;104(3):597-608.

2. Pimenta CA, Koiozumi MS, Teixeira MJ. Pain, depression, and cultural concepts. Arq Neuropsiquiatr 1997;55(3A):370-80.

3. Carneiro R, Falcone E. Um estudo das capacidades e deficiências em habilidades sociais na terceira idade. Psicologia em Estudo 2004:9(1):119-26.

4. Trentini C. qualidade de vida em idosos. Tese de Doutorado. UFRG, RS 2004.

5. Camarano A. Idosos Brasileiros: Indicadores de condições de vida e acompanhamento de políticas. Subsecretaria de Recursos Humanos. Brasília, DF; 2009. p. 6.

6. Sousa FAEF. Dor: o quinto sinal vital. Rev Latino-
-Am Enfermagem 2002:10(3):446-7.

7. Miceli AVP. A dor crônica e subjetividade em oncologia. Revista Brasileira de Cancerologia 2002:48(3):363-73.

8. Dwork R. As medidas de desfecho para ensaios clínicos de dor crônica: Recomendações IMMPACT. Dor 2005:113:9-19.

9. King T. The definition and assessment of pain. American Colege of Nurse- Midwives 2004:49(6):471-2.

10. Lung FW, Huang YL, Shu BC, et al. Parenteral rearing style, premorbid personality, mental health and quality of life in chronic regional pain: A causal analysis. Compr Psychiatry 2004:45(3):206-12.

11. Fleck MPA, Leal OF, Louzada S, et al. Desenvolvimento da versão em português do instrumento de qualidade de vida da OMS (WHOQL-100). Rev Bras Psiquiatr 1999:21(1):19-28.

12. Fleck M, Louzada S, Xavier M, et al. Application of the Portuguese version of the abbreviated instrument of quality life WHOQOL-bref. Rev Saude Publica 2000:34(2):178-83.

13. Albuquerque S. Envelhecimento Ativo: desafio dos serviços de saúde para melhoria da qualidade de vida dos idosos. Tese de Doutorado. USP, SP 2005.

Apresentado em 28 de fevereiro de 2011. Aceito para publicação 02 de junho de 2011. 